\title{
AGRESIÓN Y VIOLENCIA EN LA ESCUELA COMO FACTOR DE RIESGO DEL APRENDIZAJE ESCOLAR*
}

\author{
AGGRESSION AND VIOLENCE IN SCHOOL AS A RISK FACTOR OF \\ SCHOOL EDUCATION
}

\author{
PATRICIA CID H.**,ALEJANDRO DÍAZ M***,MARIA VICTORIA \\ PÉREZ. ${ }^{* * * *}$, MATILDE TORRUELLA $P^{* * * * *}$, Y MILADY VALDERRAMA A.*******
}

\begin{abstract}
RESUMEN
Un problema actual y creciente de salud en la comunidad escolar es la agresión y violencia observada entre los estudiantes, siendo de tal intensidad que ha provocado incidentes negativos en niños y adolescentes, como dificultad en el aprendizaje y abandono escolar, observándose esta problemática transversalmente en diversos contextos culturales y sociales. Esta revisión bibliográfica tiene como objetivo apreciar como se da la agresión y violencia entre los escolares, los factores que están involucrados en estos eventos, así como también identificar algunas intervenciones que se han llevado a cabo para prevenir y tratar estas conductas, y los resultados obtenidos. Dentro de los factores que se relacionan con la agresión escolar están los de tipo individual, familiar, escolar y del ambiente. Las intervenciones realizadas han tenido como foco a los padres, profesores y/o alumnos(as), obteniéndose resultados positivos en aquellas con enfoque integral.
\end{abstract}

Palabras Claves: Agresión, violencia, riesgo escolar, intervención, enfermería

\begin{abstract}
A current problem and increasing health in the school community is the observed aggression and violence among students, being of such intensity that has led to adverse incidents in children and adolescents, such a difficulty in learning and school dropout, having this problem across various cultural and social contexts. This bibliographical revision has the objective to appreciate how the aggression and violence occurs among students, the factors that are involved in these events, as well as to indentify some interventios that have been carried out to prevent and treat these conducts and results. Within the factors that are related with the school aggression are: those of individual type, familiar, school activities and school atmosphere. The interventions made the parents responsibles, teachers and students in general, the results of these objectives has been positive.
\end{abstract}

Key words: Aggression, violence, scholar risk, interventions, nursing

Fecha Recepción: 03 agosto 2007 Fecha Aceptación: 30 septiembre 2008

*Este trabajo forma parte del Proyecto FONDECYT No1040622 denominado "Mecanismos de riesgo y protectores asociados a los procesos de abandono y permanencia en el sistema educacional"

${ }^{* *}$ Enfermera. Magíster en Enfermería. Departamento de Enfermería. Facultad de Medicina. Universidad de Concepción. Chile.patcid@udec.cl

${ }^{* * *}$ Psicólogo. Doctor en Psicología. Departamento de Psicología. Facultad de Ciencias Sociales. Universidad de Concepción. Chile. adiazm@udec.cl

${ }^{* * * *}$ Psicóloga. Doctora en Psicología. Departamento de Psicología. Facultad Psicología. Universidad de Concepción. Chile.marperz@udec.cl

${ }^{* * * * * *}$ Enfermera. Magíster en Educación. Departamento de Enfermería. Facultad de Medicina. Universidad de Concepción. Chile.mtorruel@udec.cl

******Enfermera. Magíster en Enfermería. Departamento de Enfermería. Facultad de Medicina. Universidad de Concepción. Chile. mvalderrama@udec.cl 


\section{INTRODUCCIÓN}

Un problema actual y creciente de salud en la comunidad escolar es la agresión y violencia observada entre los estudiantes, siendo de tal intensidad que ha provocado incidentes negativos en niños y adolescentes, como dificultad en el aprendizaje y abandono escolar, observándose esta problemática transversalmente en diversos contextos culturales y sociales.

La presente revisión bibliográfica nace como inquietud frente a las respuestas emitidas por los niños, que cursaban su primer año básico de educación formal, a una entrevista estructurada que formó parte de una investigación longitudinal cuyo propósito era identificar los mecanismos o factores de riesgo y protectores asociados a los procesos de abandono y permanencia en el sistema educacional. Se preguntó a los niños que les gustaba y que no les gustaba de sus profesores, de sus compañeros y de su escuela. En ambos tipos de preguntas las respuestas fueron muy similares repitiéndose con frecuencia las siguientes frases: "me pegan", "me aíslan", "se burlan".

Diferentes estudios informan que los episodios de agresión y/o violencia en las escuelas producen en los niños daños físicos y emocionales, estrés, desmotivación, ausentismo, e incluso efectos negativos en el rendimiento escolar por estrés postraumático en los afectados (Gumpel \& Meadan 2000; Verlinde, Hersen \&Thomas 2000; Henao, 2005; Smith \& Thomas, 2000 ).

Las conductas agresivas o violentas que perciben los niños de parte de sus compañeros pueden pasar desapercibidas por el personal de la escuela, como también por algunos padres que consideran estos comportamientos típicos de la edad y que los ayudan a crecer (Rodríguez, Seoane \& Pedreira, 2006). Otra situación que dificulta la valoración del problema es el pacto de silencio entre agresores y agredido (Gumpel \& Meadan 2000; Cerezo, 2006).

De acuerdo al estudio de violencia en establecimientos educacionales en Chile, la agresión y violencia en los ambientes de es- tudio constituyen un problema que requiere una pronta intervención. De este estudio, un $45 \%$ de los estudiantes señaló haber sido agredido y, a su vez, el 38\% declaró ser agresor. La mayoría de los estudiantes de 10 a 13 años percibió agresión psicológica, como ser ignorados, recibir insultos o garabatos, burlas, descalificaciones, gritos y rumores mal intencionados en los espacios de recreos y de deportes (Ministerio de Educación [MINEDUC], 2006).

Las conductas de agresión y violencia alteran el ambiente escolar repercutiendo negativamente en el aprendizaje. Un buen clima escolar que favorece el aprendizaje estaría definido por tres factores: no violencia, ausencia de perturbaciones para estudiar y amistad (Ascorra, Arias \& Graff, 2003).

Esta revisión bibliográfica tiene como objetivo apreciar como se da la agresión y violencia entre los escolares, los factores que están involucrados en estos eventos, así como también identificar algunas intervenciones que se han llevado a cabo para prevenir y tratar estas conductas, y sus resultados.

\section{¿Que se entiende por conflicto, agresión, agresividad y violencia?}

"Los conflictos son situaciones en que dos o más personas entran en oposición o desacuerdo de intereses y/o posiciones incompatibles donde las emociones y sentimientos juegan un rol importante..."(Unidad de Apoyo a la Transversalidad, 2006, p. 12). La agresión y la violencia son conflictos, "la agresión es una respuesta hostil frente a un conflicto latente, patente o crónico", y la violencia se asocia a un conflicto "en el que no se sabe cómo regresar a una situación de orden y respeto de las normas sociales" (Unidad de Apoyo a la Transversalidad, 2006, p. 14).

Maturana refiere que las emociones influyen en que una acción sea de agresión o de una caricia, desde esa perspectiva conceptualiza la agresión como la emoción a través de la 
cual el otro es negado directa o indirectamente como un legítimo otro en coexistencia con uno (In: Comité Paulista para a década da cultura da Paz, 2003).

Para Oteros (2006) la conducta agresiva es socialmente inaceptable ya que puede llevar a dañar física o psicológica a otra persona, la agresividad en la etapa escolar puede aplicarse a acciones agresivas (conductas), a estados de ánimo (sentimientos subjetivos), a impulsos, pensamientos e intenciones agresivas, y a las condiciones en que es probable que se adopten conductas agresivas (estimulación ambiental).

Pintus (2005) conceptualiza la violencia escolar como una manifestación que se da en el espacio de las relaciones humanas en el contexto de las instituciones educativas. Las consecuencias vivenciales de esta violencia son negativas, como sentirse lastimado, dañado, despreciado, menospreciado, disminuido, y/o maltratado.

Algunos autores identifican que los factores que inciden en que un niño sea agresivo o violento, o esté en riesgo de desarrollar estas conductas, son diversos (Henao, 2005; Verlinde et al., 2000). Así agrupan los factores de riesgo en tres ámbitos: personales, de su familia y del ambiente escolar.

Sin embargo, Verlinde et al. (2000), al realizar una revisión de la literatura sobre causas y correlaciones de la violencia entre los niños, reconocen además de los aspectos individuales, familiares, escuela/pares, el aspecto societario/ambiental y esquematizan muy bien los factores de riesgo para la violencia escolar (Tabla 1). Estos autores tienen presente en su estudio, lo expuesto por Pepler y Slaby (1994), que la agresión entre las niñas se desarrolla y se expresa en forma diferente (In: Verlinde et al., 2000).

Tabla 1. Factores de riesgo para violencia escolar de Verlinde, Hersen y Thomas (2000).

\begin{tabular}{|c|c|c|c|}
\hline Individual & Familiar & Escuela/Pares & Societario/ambiental \\
\hline $\begin{array}{l}\text { Condición médica / } \\
\text { física }\end{array}$ & Monitoreo pobre & $\begin{array}{l}\text { Grupo de pares } \\
\text { antisociales }\end{array}$ & Pobreza \\
\hline Temperamento & Exposición a & Bajo compromiso & Desorganización del \\
\hline difícil & violencia & de la escuela & vecintenario \\
\hline $\begin{array}{l}\text { Impulsividad / } \\
\text { hiperactividad }\end{array}$ & $\begin{array}{l}\text { Abuso del } \\
\text { niño/negligencia }\end{array}$ & Fracaso académico & Violencia del entorno \\
\hline $\begin{array}{l}\text { Condiciones } \\
\text { psiquiátricas }\end{array}$ & $\begin{array}{l}\text { Abuso parental de } \\
\text { sustancias }\end{array}$ & Escuelas grandes & Acceso a las armas \\
\hline Historia de agresión & Paternidad inefectiva & Involucración en pandillas & Prejuicios \\
\hline Abuso de sustansias & Conflicto marital & Aislamiento social & Expectativas de rol de genero \\
\hline Actitudes/creencias & Pobre apego & Rechazo de pares & Normas culturales \\
\hline Narcisismo & Padres antisociales & Intimidación (Bullying) & \\
\hline
\end{tabular}

\section{Características personales del niño}

Los niños aproximadamente hasta los siete años de edad obedecen a los adultos sin cuestionar las reglas impuestas, de tal manera que consideran que un acto es correcto o incorrecto y que un acto incorrecto merece castigo. Después de los 11 años desarrollan la capacidad para razonar moralmente, las reglas son modificables y evalúan situaciones específicas para su aplicación (Papalia, Wendkos \&Duskin, 2005a).

Los adultos han confundido las interacciones agresivas entre los niños con juegos propios de su edad, como se mencionó anteriormente, lo que ha llevado a una distorsión de la realidad (Papalia, Wendkos \& Duskin, 2005b). Es importante diferenciar las conduc- 
tas agresivas del juego, de la agresión y violencia propiamente tal, para poder identificar la dimensión de este problema y tomar medidas para su tratamiento y prevención (Gumpel \& Meadan, 2000).

En otros estudios se ha observado que los niños que se caracterizan por ser irritables, con bajo autocontrol, muy activos y con problemas de atención e impulsividad presentan más probabilidades de mostrar problemas de conducta y conducta antisocial que los niños que no presentan tales características (Ayala, Pedroza, Morales, Chaparro y Barragán, 2002). En el mismo sentido De la Barra, Toledo y Rodríguez (2003) encontraron que los escolares catalogados por los profesores como agresivos/desobedientes en su primer año escolar persistieron con este tipo de conductas seis años después.

\section{Ambiente familiar}

Para algunos investigadores los problemas de conducta y rendimiento escolar del niño evidencian los conflictos de sus padres (Ruiz \& Gallardo, 2002; Jadue, 2002). La modernización ha impactado en la estructura y función de las familias chilenas, afectando la socialización de los hijos y su educación (Jadue, 2003).

Jadue (2003) indica que los cambios en la familia, cómo familias con un solo padre o familias disfuncionales, constituyen un riesgo inminente que se suma a otros factores dañinos que pueden afectar la educación chilena. El efecto de ello se observa en un menor rendimiento escolar, en el abandono del sistema educativo, en las manifestaciones conductuales desadaptativas y en las expresiones emocionales negativas de los niños.

Ruiz y Gallardo (2002) observaron en su estudio que los hijos/as de familias negligentes manifestaban poca adaptación general en el aspecto psicológico, inferior rendimiento escolar y mayor distracción en el aula. También se señala que un niño o niña con abandono familiar le será más difícil manejar los traumas en la etapa adulta (Marty \& Carvajal, 2005).

Familias disfuncionales incompletas, con manifestaciones de agresividad, mala integración social y familiar, rechazo e irresponsabilidad en el cuidado y atención de sus hijos y con presencia de alcoholismo, fueron características de las familias de niños de 9 a 11 años diagnosticados con conductas agresivas (Noroño, Cruz, Cadalso \& Fernández, 2002).

En cuanto a los hijos/as pertenecientes a familias que presentan violencia intrafamiliar, un estudio realizado en Nicaragua muestra que un 63\% de los hijos de familias con este problema repiten años escolares o abandonan la escuela en promedio a los nueve años de edad, y son tres veces más propensos a asistir a consultas médicas (Organización Panamericana de la Salud [OPS], 1998).

Se han observado efectos perdurables en el desarrollo de los niños y niñas que viven en hogares violentos. Ellos y ellas pueden presentar en el futuro, pocas habilidades sociales y conductas agresivas, de tal manera que los niños que han sufrido violencia durante su infancia, serán adultos agresores (Fondo de las Naciones Unidas [UNICEF], 2006). Aprehendiendo esta conducta y repitiéndola, dañando con ello a sus seres queridos, y a los más vulnerables dentro de la familia: sus hijos e hijas.

Desde el punto de vista de los factores protectores de la familia asociados a una menor agresión del niño es importante destacar la organización familiar, la orientación a alcanzar metas, la tendencia a la religiosidad, y la cohesión familiar (Ayala et al., 2002).

\section{Ambiente escolar}

Los niños en un estudio realizado en escolares de segundo básico declararon que les gustaba ir a la escuela para aprender, pero les disgustaba el desorden y la violencia de sus compañeros (Valderrama et al., 2007). 
Maturana y Dávila (2006) expresan que la educación es fundamental "pero no en términos de aprendizaje de materias, sino en términos de convivencia". Para Maturana (2001) es primordial enseñar a un niño/a a respetarse y aceptarse, sólo así aprenderá a respetar y aceptar a sus compañeros y vivir en armonía con su entorno. Los niños tienen que aprender a ser, aprender a hacer, aprender a aprender y aprender a convivir. En la escuela el niño aprende sobre la vida y aprende a convivir, siempre que este ambiente sea propicio para desarrollar estas capacidades.

Las interacciones sociales con sus profesores y compañeros son de suma importancia para el desarrollo académico y social del niño, las opiniones que recibe de ellos le condicionan positiva o negativamente sobre su valía personal, lo que repercutirá posteriormente en su motivación y rendimiento académico (García \& Doménech, 1997).

Freire (1997) señalaba que "si se respeta la naturaleza del ser humano, la enseñanza de los contenidos no puede darse alejada de la formación moral de los educandos" (p. 34). Este autor considera que si los profesores van guiando a los niños en la construcción de su conocimiento formarán seres humanos libres, justos y equitativos, para desenvolverse en la sociedad. Del mismo modo, si los profesores fomentan las buenas relaciones interpersonales, el trabajo en equipo, la amistad, entre otras, contribuyen al desarrollo de la empatía, de prácticas de aprendizaje cooperativo, incremento de la motivación escolar y la participación de los educandos en el proceso educativo (Guil \& Mestre, 2004).

Verlinde et al. (2000) observaron que los niños que tuvieron profesores que mantenían el orden en la sala de clases y proporcionaban claras guías para una conducta aceptable, mostraron menos agresión en los cursos superiores. En cambio, los que tuvieron un profesor débil y un ambiente caótico presentaron mas agresión en sus otros años de estudios y tendieron a formar o reunirse más con grupos antisociales.
En el estudio de Francia (2003) los niños indisciplinados manifestaron maltrato físico y psicológico por parte de sus educadores, ellos presentaron factores de riesgo como desatención, carencia afectiva, violencia familiar, entre otros; lo que hacía que los niños fueran de difícil manejo, e irremediablemente maltratados por sus profesores. Conducta que no soluciona la indisciplina y agrava la situación, al sancionar y castigar se promueve la violencia y el desamor (Maturana \& Dávila, 2006).

Entre los hallazgos del Primer Estudio de Convivencia Escolar desarrollado en Chile, llamó la atención la poca consideración que refirieron los alumnos a la resolución de problemas que se plantean en el centro educacional, y que alteran la convivencia escolar (Instituto de Evaluación y Asesoramiento Educativo, 2005).

Para Cerezo (2006) se está viviendo un fenómeno en las escuelas llamado bullying, o agresiones sistemáticas entre compañeros en las aulas, está dinámica de agresión y victimización no se lleva a cabo frente a los adultos y sólo se hace visible cuando constituye un problema mayor. Los agresores o bullies actúan "movidos por un abuso de poder y un deseo de intimidar y dominar, mientras que el alumno víctima se encuentra indefenso" (Cerezo, 2006 p. 27).

Los bullies y las víctimas, comúnmente son niños, y muestran escaso autocontrol en sus relaciones sociales: Los bullies son más grandes que sus compañeros y más fuertes, se creen líderes, sinceros, con alta autoestima, con actitud negativa hacia la escuela y con bajo rendimiento escolar, en cambio, las víctimas se consideran tímidos, con menor fuerza física, y de baja ascendencia social (Cerezo, 2001).

\section{Ambiente social}

Los niños continuamente están recibiendo mensajes dañinos de su entorno. Por ejem- 
plo, en los contenidos de sus asignaturas se valoran las guerras, muchos de sus familiares resuelven sus conflictos con gritos o insultos, en la televisión las noticias que impactan son de violencia, igualmente las de otros medios de comunicación. Se ha observado efectos negativos de la violencia televisiva sobre la cognición, la emoción y la conducta infantil (Pérez-Olmos, Pinzón, González-Reyes \& Sánchez-Molano, 2005). Los niños imitan a sus familiares o héroes televisivos, y expresan sus emociones negativas con golpes y ofensas hacia los otros.

\section{Intervenciones}

Jadue (2003) indica que el rol de la escuela es fundamental en el desarrollo personal y valórico de los niños, expresa que hay que enseñar a los niños a sobrellevar las vicisitudes de la vida, trabajar con los recursos personales internos de los alumnos, logrando así disminuir riesgos de déficit educacional y de deserción escolar.

Positivo fueron los resultados de un programa de intervención educativa en padres con hijos diagnosticados con conductas agresivas, destinado a modificar los modelos educativos agresivos en la familia. Después de cuatro meses de aplicación del programa de intervención educativa, la responsabilidad de los padres en la educación y cuidado del niño aumentó y se redujeron las manifestaciones de agresividad en el medio familiar (Cruz, Noroño, Fernández, \& Cadalso, 2002).

Shapiro (1997) afirma que los juegos cooperativos han demostrado disminuir significativamente la ira y la agresión entre los niños. Al evaluar los dos primeros años del programa "juego del buen comportamiento (JBC)" aplicados a niños de primeros básicos, se observó que los niños diagnosticados al inicio del programa con conductas de timidez, agresión, hiperactividad o inmadurez emocional no presentaron cambios durante el primer año, pero sí disminuyeron estas conductas en el segundo año de intervención (Pérez, Rodríguez, De la Barra \& Fernández, 2005). Los niños sin conductas disruptivas ni agresivas que participaron en el JBC no modificaron su conducta durante el primer ni segundo año de aplicación del programa (Pérez, et al., 2005). Es importante apreciar que este programa se puede aplicar a niños y niñas con o sin alteración de la conducta, pues ellos aprenden normas en forma entretenida compartiendo con sus compañeros.

Un esquema para la eliminación de la violencia propuesto por Martínez-Otero (2005) considera que directivos, profesores, trabajadores del sistema escolar, niños, padres y familias son parte de la comunidad educativa y es su responsabilidad participar en resolver y prevenir la violencia escolar (Tabla 2).

Tabla 2. Esquema para la eliminación de la violencia, adaptado de Martínez-Otero (2005).

\begin{tabular}{|c|l|}
\hline Análisis de la situación & $\begin{array}{l}\text { Establecer un diagnóstico rápido y certero del problema, de los sujetos } \\
\text { y de su circunstancia. }\end{array}$ \\
\hline Bases teóricas de la intervención & $\begin{array}{l}\text { Utilizar un enfoque humanistico abierto e integrador. En el caso de las } \\
\text { intervenciones individuales, enriquecer esta perspectiva con técnicas } \\
\text { cognitivo-conductuales que posibiliten la modificación de pensamientos, } \\
\text { de motivaciones y de actitudes, combinarse siempre con el trabajo } \\
\text { comunitario }\end{array}$ \\
\hline
\end{tabular}




\begin{tabular}{|c|c|}
\hline Planificación de la intervención. & $\begin{array}{l}\text { La intervención puede requerir la participación de toda la comunidad } \\
\text { educativa. Desde una perspectiva ecológica y sistémica, se considera muy } \\
\text { importante la adaptación del programa de prevención y de resolución } \\
\text { de conflictos a las características concretas del centro. }\end{array}$ \\
\hline $\begin{array}{l}\text { Buscar la implicación de toda la } \\
\text { comunidad educativa. }\end{array}$ & $\begin{array}{l}\text { Hay que procurar que las personas que integran la institución tomen } \\
\text { parte en distintos acontecimientos enriquecedores del ambiente. Como } \\
\text { por ejemplo aniversarios, graduaciones, etc. }\end{array}$ \\
\hline La promoción de la convivencia & $\begin{array}{l}\text { Es necesaria una estructura que vertebre el clima social, y que sea lo } \\
\text { suficientemente flexible como para admitir cambios }\end{array}$ \\
\hline $\begin{array}{l}\text { El establecimiento de normas } \\
\text { claras. }\end{array}$ & $\begin{array}{l}\text { La convivencia precisa de un reglamento fundado en la razón. Un código } \\
\text { que regule el comportamiento, y en el que queden recogidos los derechos y } \\
\text { los deberes de las personas. Las normas deben ser realistas y consensuadas; } \\
\text { de lo contrario, se viven como imposiciones que hay que transgredir. }\end{array}$ \\
\hline $\begin{array}{l}\text { El estudio de cada caso y la } \\
\text { atención personal, aspectos a } \\
\text { considerar. }\end{array}$ & $\begin{array}{l}\text { Adoptar medidas grupales, también es pertinente ayudar a cada educando } \\
\text { en función de su singularidad. Actualmente esto es insuficiente, porque } \\
\text { se centra en la dimensión intelectual y en el rendimiento académico, es } \\
\text { oportuno completarla con el cultivo de la dimensión socioafectiva. }\end{array}$ \\
\hline Coherencia del currículo. & $\begin{array}{l}\text { Las programaciones tienen que incluir de modo explícito aspectos relativos } \\
\text { a los desarrollos social, afectivo y ético. }\end{array}$ \\
\hline Evaluación Final & $\begin{array}{l}\text { Evaluar clima social, desarrollo moral, trama comunicativa, etc. Con la } \\
\text { participación de expertos }\end{array}$ \\
\hline
\end{tabular}

Los adultos que son parte del ambiente escolar deben pesquisar la intensidad y frecuencia de las agresiones entre los escolares y establecer una disciplina consistente que vaya a solucionar este problema. Las vías específicas favorecedoras de la convivencia escolar son: la disciplina, la negociación, la mediación y el fomento de la sana competencia social (Martínez-Otero, 2005).

Es necesario comunicarse con niños y niñas y determinar que tipo de agresión recibe o realiza el niño y considerar los factores de riesgo, sean estos personales, familiares, escolares y/o situacionales, para poder intervenir a tiempo y facilitar su normal desarrollo infantil (Verlinde et. al, 2000).

La familia y la escuela tienen responsabilidades en la educación de los niños, estableciendo una comunicación escuela-familia, donde la escuela sea un espacio abierto a las familias de los alumnos y de sus profesores, facilitando la socialización de los niños (Rivera y Milicic, 2006; Kliksberg, 2005).

Es importante reconocer el rol que el equipo de la salud tiene en las diferentes etapas de la intervención que se señalan en el esquema de Martínez-Otero. Estos equipos funcionan en los Centros de Salud Familiar y mantienen una estrecha comunicación con los establecimientos educacionales y las familias de los escolares, de tal manera que su participación se debe observar desde la primera etapa de intervención. Un programa eficiente del equipo de salud, y en especial del profesional de enfermería, se orienta a la promoción de un ambiente saludable en la comunidad escolar, y a intervenciones en los diferentes niveles de prevención del problema de agresión y violencia escolar. 
En el nivel de prevención secundaria en salud, se establece un diagnóstico de riesgo de violencia en base a indicadores, tales como los señalados en el esquema de Martínez-Otero. Este diagnóstico se enriquece y permite visualizar la problemática a los actores, si se realiza en forma participativa con los estamentos de la comunidad escolar (profesores, padres, escolares y administrativos).

La intervención precoz e integral permitirá solucionar o detener el avance de la violencia en situaciones de complejo manejo. Con ello se evita en gran medida el efecto negativo de la violencia en el progreso académico de los escolares y el abandono del sistema educativo, que en muchas ocasiones promueve en estas personas conductas antisociales.

Una interesante forma de trabajo factible de ser aplicada por profesionales del área de la salud, y en particular de Enfermería, es el modelo ecológico propuesto por la Organización Panamericana de la Salud, que aborda acciones a nivel (1) individual, (2) de las relaciones del grupo más cercano, (3) de la comunidad y (4) del microsistema escolar (Concha-Eastman, 2008; OPS - Ministerio Federal de Cooperación Económica y Desarrollo (GTZ), 2008).

El modelo ecológico propuesto por la OPSGTZ, plantea acciones a nivel individual, de las relaciones más cercanas, comunitario y nivel social. En los niveles individual y familiar, puede ser aumentar el apoyo mediante visitas domiciliarias a familias con hijos en edad escolar y en situación de riesgo. Así como en los controles de salud escolar detectar características que hagan sospechar la presencia de niños/as agredidos o agresores, o violencia intrafamiliar. Especialmente, llevar a cabo entrevistas con los profesores y las profesoras para planificar actividades educativas y de intervención en equipo.

A nivel de las comisiones de Educación y Salud es posible efectuar abordaje interdisciplinario e intersectorial para prevenir, tratar y rehabilitar este problema.

\section{CONSIDERACIONES FINALES}

Existen conflictos, conductas agresivas y violentas en los niños que asisten a establecimientos educacionales básicos, afectando las relaciones interpersonales y por ende el ambiente escolar. Este fenómeno se asocia a diversos factores tanto del niño, como de su familia, de su entorno escolar y social.

Para disminuir este problema, es necesaria la participación de la comunidad escolar en establecer normas de respeto en los establecimientos escolares que incluyan las relaciones entre alumnos/as, profesores/as, apoderados/ as, directivos/as, administrativos/as y personal de salud escolar. Es importante llegar a un consenso sobre la disciplina que se impondrá sobre ciertos actos que dañan a los niños, socializándolos con las familias y sus hijos que integran la comunidad escolar.

Los equipos de salud familiar y dentro de ellos el profesional de enfermería, tiene la posibilidad de establecer estrategias de acción frente a los niños y familias en situación de riesgo de violencia.

Es responsabilidad de todas las personas que interactúan en la comunidad escolar participar en acciones que favorecen la convivencia escolar: pesquisando las conductas agresivas e identificando a agresores y víctimas para establecer medidas protectoras y tratamientos oportunos, estableciendo una comunicación permanente con los niños, fomentando el respeto y creando ambientes agradables para el aprendizaje, educando con afecto y firmeza. Sólo con la participación de toda la comunidad escolar se puede prevenir y/o tratar este problema que afecta la salud y la educación de los niños.

\section{REFERENCIAS BIBLIOGRÁFICAS}

Ascorra, P., Arias, H., \& Graff, C. (2003). La escuela como contexto de contención social y afectiva. Revista Enfoques Educacionales, 5(1), 117-135. 
Ayala, H., Pedroza, F., Morales, S., Chaparro, A., y Barragán, N. (2002). Factores de riesgo, factores protectores y generalización del comportamiento agresivo en una muestra de niños en edad escolar. Salud Mental, 25(3), 27-40.

Cerezo, F. (2006). Análisis comparativo de variables socio-afectivas diferenciales entre lo implicados en bulling. Estudio de un caso de víctima-provocador. Anuario de Psicología Clínica y de la salud, 2, 27-34.

Cerezo, F. (2001). Variables de personalidad asociadas en la dinámica bullying (agresores versus víctimas) en niños y niñas de 10 a 15 años. Anales de Psicología, 17(1), 37-43.

Comité Paulista para a década da cultura da Paz (2003). Las contribuciones de Humberto Maturana a las Ciencias de las Complejidades y a la Psicología. Un Programa da UNESCO 20012010. Recuperado el 8 de marzo de 2007 de http://www.comitepaz.org.br/Maturana4 3. $\underline{\mathrm{htm}}$

Concha-Eastman, A. (2008). Contribución de OPS, prevención de violencia - enfoque de Salud Pública. Recuperado el 31 de julio de 2008 de http://scm.oas.org/pdfs/2008/ CP20720T01.ppt.

Cruz, R., Noroño, N., Fernández, O., \& Cadalso, R. (2002). Intervención educativa para la disminución de la agresividad en la conducta infantil. Revista Cubana de Pediatría, 74(3), 189-194.

De La Barra, F., Toledo, V \& Rodríguez, J. (2003). Estudio de salud mental en dos cohortes de niños escolares de Santiago Occidente. III: Predictores tempranos de problemas conductuales y cognitivos. Revista Chilena de Neuropsiquiatría, 41(1), 65-76.

Francia, ME. (2003). Maltrato infantil: Un problema de todos. Revista Cubana de Medicina General Integral, 19, (1). Recuperado el 16 de febrero de 2007 de http://scielo.sld.cu/scielo. php? script $=$ sci_arttext \&pid $=$ S0864 -2125200 3000100009\&lng=es\&nrm=iso\&tlng=es

Freire, P. (1997). Pedagogía de la autonomía, Cap. 1, No hay docencia sin discencia. México: Editorial Siglo XXI.

García, F. \& Doménech, F. (1997). Motivación, aprendizaje y rendimiento escolar Revista Electrónica de Motivación y Emoción, 1, 1-16.

Guil, R., \& Mestre J. (2004). Violencia escolar: su relación con las actitudes sociales del alumna- do y el clima social del aula. : Revista electrónica Iberoamericana de psicología social 2, (1). Recuperado el 4 de marzo de 2007 de http://dialnet. unirioja.es/servlet/articulo? codigo $=1088456$

Gumpel, TP. \& Meadan, H. (2000). Children's perceptions of school-based violence. British Journal of Educational Psychology, 70, (3), 391-404.

Henao, J. (2005). La prevención temprana de la violencia: una revisión de programas y modalidades de intervención.Universitas Psychologica, 4(2), 161-177.

Instituto de Evaluación y Asesoramiento Educativo (2005). Primer Estudio de Convivencia Escolar. Recuperado el 5 de marzo de 2007 de http://innovemos.unesco.cl/medios/DOC/ DyC/Estudios/resumenejecutivo.pdf

Jadue, G. (2002). Factores psicológicos que predisponen al bajo rendimiento, al fracaso y a la deserción escolar. Estudios pedagógicos, 28, 193-204.

Jadue, G. (2003). Transformaciones familiares en Chile: riesgo creciente para el desarrollo emocional, psicosocial y la educación de los hijos. Estudios pedagógicos, 29, 115-126.

Kliksberg, B. (2005). La familia en América Latina, realidades, interrogantes y perspectivas. Convergencia, 12(38), 13-41.

Maturana, H., y Dávila, X. (2006). Desde la matriz biológica de la existencia humana. Revista PRELAC, 2, 30-39.

Maturana, H. (2001). Emociones y lenguaje en educación y política, Cap. 1, Una mirada a la educación actual desde la perspectiva de la biología del conocimiento. Décima ed. Santiago: Editorial Dolmen Ensayo.

Martínez-Otero V. (2005). Conflictividad escolar $\mathrm{y}$ fomento de la convivencia. Revista Iberoamericana de Educación, 38, 33-52.

Marty, C. \& Carvajal, C. (2005). Maltrato infantil como factor de riesgo de trastorno por estrés postraumático en la adultez. Revista chilena de neuro-psiquiatría, 43(3), 180-187.

MINEDUC (Ministerio de Educación). (2006). Estudio nacional de violencia en establecimientos educacionales en Chile. Recuperado el 3 de julio de 2007 de http://www.educarchile. cl/UserFiles/P0001/File/CR Articulos/Presentacion $\% 20$ Estudio $\% 20$ Nacional $\% 20 \mathrm{de} \% 20$ Violencia\%20Escolar 2006.pdf

Noroño, N., Cruz, R., Cadalso, R., \& Fernández, O. 
(2002). Influencia del medio familiar en niños con conductas agresivas. Revista Cubana de Pediatría, 74(2), 138-144.

Oteros, AM. (2006). La agresividad como conducta perturbadora en el aula. Revista Digital Investigación y Educación, 26, (3). Recuperado el 3 de julio de 2007 de http://www.csicsif.es/ andalucia/modules/mod_sevilla/archivos/ revistaense/n26/26080151.pdf

Organización Panamericana de la Salud.(OPS) (1998). Presentation of primilary results of multicountry violence study, En La violencia contra la mujer en las Américas. Recuperado el 15 de noviembre del 2006 de http://www.paho. org/spanish/gov/ce/spp/spp31 6.pdf

Organización Panamericana de la Salud. OPS Ministerio Federal de Cooperación Económica y Desarrollo. GTZ (2008). ¡Preparados, listos, ya! Una síntesis de intervenciones efectivas para la prevención de violencia que afecta a adolescentes y jóvenes. Washington: OPS.

Papalia, D., Wendkos, S. \& Duskin, R. (2005a). Psicología del desarrollo. De la Infancia a la adolescencia, Cap. 13, Desarrollo cognoscitivo en la niñez intermedia. Novena ed. México: Editorial McGraw-Hill.

Papalia, D., Wendkos, S. \& Duskin, R. (2005b). Psicología del desarrollo: De la Infancia a la adolescencia, Cap.11, Desarrollo psicosocial en la niñez temprana. Novena ed. México: Editorial McGraw-Hill.

Pérez-Olmos, I., Pinzón, A., González-Reyes, R. \& Sánchez-Molano, J. (2005). Influencia de la televisión violenta en niños de una escuela pública de Bogotá, Colombia. Revista de Salud Pública, 7(1), 70-88.

Pérez, V., Rodríguez, J., De la Barra, F. \& Fernández, AM. (2005). Efectividad de una estrategia conductual para el manejo de la agresividad en escolares de enseñanza básica. Psykhe, 14(2), 55-62.
Pintus, A. (2005). Violencia en la escuela: compartiendo la búsqueda de soluciones. Revista Iberoamericana de Educación, 37, 117-34.

Rodríguez, R., Seoane, A. \& Pedreira JL. (2006). Niños contra niños: el bulling como trastorno emergente. Anales de Pediatría, 64(2), 162166.

Rivera, M., \& Milicic, N. (2006). Alianza FamiliaEscuela: Percepciones, Creencias, Expectativas y Aspiraciones de Padres y Profesores de Enseñanza General Básica. Psykhe, 15(1), 119-135.

Ruiz, I. \& Gallardo, JA. (2002). Impacto psicológico de la negligencia familiar (leve versus grave) en un grupo de niños y niñas. Anales de Psicología, 18(2), 261-272.

Shapiro, L. (1997). La inteligencia emocional de los niños, Cap. 4 , La capacidad de resolver problemas. Bilbao, España: Grupo Zeta.

Smith, H. \& Thomas, S. (2000). Violent and nonviolent girls: contrasting perceptions of anger experiences, school, and relationships. Mental Health Nursing, 21, 547-75.

Fondo de las Naciones Unidas UNICEF. (2006). Los más pequeños, entre las mayores víctimas de la violencia doméstica. Recuperado el 15 noviembre 2006 de http://www.unicef.org/ spanish/media/media 35151.html

Unidad de Apoyo a la Transversalidad. (2006). Conceptos clave para la resolución pacífica de conflictos, en el ámbito escolar. Cartilla de trabajo aprender a convivir (Inscripción No157.007-2006). Santiago: Ministerio de Educación de Chile.

Valderrama, M., Behn, V., Pérez, MV., Díaz, A., Cid, P. \& Torruella, M. (2007). Factores de riesgo biopsicosocial que influyen en el fracaso escolar en alumnos vulnerables de escuelas municipalizadas de la comuna de San Pedro de la $\mathrm{Paz}, 2005$. Cienc. enferm., 13(2), 41-52.

Verlinde, S., Hersen, M. \& Thomas, J. (2000). Risk factors in school shootings. Clinical Psychology Review, 20 (1), 3-56. 